$\checkmark$ Research Square

\title{
Cannabidiol promotes neurogenesis in the dentate gyrus during an abstinence period in rats following chronic exposure to methamphetamine
}

\section{Yasaman Razavi}

Cellular and Molecular research center,Iran University of Medical Science

Fariborz Keyhanfar

Iran University of Medical Sciences: Tehran University of Medical Sciences

Abbas Haghparast

Shahid Beheshti University of Medical Sciences Neuroscience Research Center

Ronak Shabani

Iran University of Medical Sciences

Mehdi Mehdizadeh ( $\nabla$ mehdizadeh.m@iums.ac.ir)

Iran University of Medical Sciences: Tehran University of Medical Sciences https://orcid.org/00000002-9268-7318

\section{Research Article}

Keywords: Neurogenesis, Methamphetamine, Cannabidiol, Chronic exposure, Abstinence, Dentate gyrus

Posted Date: April 19th, 2021

DOl: https://doi.org/10.21203/rs.3.rs-256868/v1

License: (9) (1) This work is licensed under a Creative Commons Attribution 4.0 International License. Read Full License 


\section{Abstract}

Chronic methamphetamine (meth) abuse can lead to certain deficits in the hippocampal function by affecting the hippocampal neurogenesis and plasticity. To determine whether cannabidiol (CBD) can promote proliferation and maturation of neuronal progenitor cells, this study investigated the CBD effect on neurogenesis in the hippocampal dentate gyrus (DG) following chronic exposure to meth in rats. The rats received $2 \mathrm{mg} / \mathrm{kg}$ of meth twice a day for ten days. Next, immunofluorescence was performed to evaluate the effect of intracerebroventricular (ICV) administration of CBD $(50 \mu \mathrm{g} / 5 \mu \mathrm{L})$ over an abstinence period (ten days) on the expression levels of neurogenesis markers, such as Ki67, NeuN, and doublecortin (DCX). Moreover, neuronal degeneration in the hippocampus was assessed using Nissl staining. According to our findings, repeated ICV administration of CBD improved cell proliferation and neurogenesis and increased the number of Ki-67 and DCX-positive cells in the abstinence period. Meanwhile, meth treatment subjects caused a significant decrease in the number of neurogenesis makers, as compared to the control group. The neurogenesis markers (Ki-67 and DCX) could be somewhat reversed, while NeuN did not show any significant increase in the CBD group. Our findings demonstrated that CBD can induce neuroprotective effects by modulating neurogenesis. Therefore, it can provide a promising therapeutic approach to improve cognitive performance following chronic exposure to psychostimulant drugs, including meth.

\section{Introduction}

Adult neurogenesis is a distinguished example of activity-dependent neuroplasticity. It is influenced by numerous factors, including stress, drugs, and brain damage (Jun et al., 2012; Parent 2003; Van Praag et al., 1999). The properties of neural progenitor cells detected in the sub-granular zone (SGZ) are shown by adult hippocampal neurogenesis of the dentate gyrus (DG). After proliferation and migration of these cells to the granular zone (GZ), they are distinguished from mature granule neurons (Kempermann, 2008). Different neurogenesis phases can be identified using specific markers, and proliferation of progenitor cells is evaluated by Ki-67 (Gerdes et al., 1984).

As a microtubule-associated protein, doublecortin (DCX) was detected in precursor cells, besides not fully formed neurons in phases of migration or differentiation. NeuN is identified as a nuclear protein only in mature neurons (Kempermann 2008). Neonatal neurons improve neural plasticity to maintain certain brain functions, such as mood regulation and spatial learning (Llorens-Martín et al., 2006; Santarelli et al., 2003). Clinical studies have shown that methamphetamine (meth) dependence alters the hippocampal morphology (Canales 2010a; Kim et al. 2010).

Numerous studies have indicated the significant effects of hippocampal plasticity or the so-called phenomenon of "adult hippocampal neurogenesis" on memory and hippocampus-dependent learning (Jessberger et al., 2009; Leuner et al., 2006; Shors et al., 2001; Van Praag et al., 1999). It is known that meth changes all phases of hippocampal neurogenesis. These effects vary based on drug dosage, drug exposure pattern, exposure duration, and drug exposure schedule before labeling neural progenitors 
(Hildebrandt et al., 1999; Mandyam et al., 2008; Teuchert-Noodt et al., 2000; Yuan et al., 2011a). The inhibitory effect of meth on the active regenerative abilities of the adult hippocampus is now distinguished as a neurodegeneration precursor, stimulated by meth in this region (Canales 2007; Canales 2010b)

Adult neurogenesis has been shown to be a solid instance of brain plasticity, modulated by the endocannabinoid system. In recent years, numerous studies have reported the expression of the endocannabinoid system in neural stem cells. The non-psychoactive cannabidiol (CBD) has become an interesting research subject in clinical and preclinical areas to treat substance abuse disorders (Wenzel and Cheer 2018). CBD dampens behavioral and molecular manifestations of maladaptive neuroplasticity, which underlies drug addiction (Fogaça et al. 2018). Besides, CBD induces several effects in the central nervous system (CNS) and dramatically affects the generation of neural progenitors in early developmental stages.

Chronic intake of psychostimulant drugs is related to cognitive deficits in attention, memory, and executive functions (Potvin et al. 2014; Santarelli et al. 2003). The improvement and treatment of these cognitive deficits can prevent drug relapse (Rezapour et al. 2016; Sofuoglu et al. 2016). Moreover, CBD has been shown to have pro-cognitive effects in preclinical studies. In a previous study on mice, pretreatment with intermediate doses of CBD $(20 \mathrm{mg} / \mathrm{kg})$ for a ten-day period facilitated the new object recognition task and enhanced the learning markers, namely, brain-derived neurotrophic factor (BDNF) expression and neural progenitor proliferation in the hippocampus (Luján et al. 2018). It also prevented memory impairment in the object recognition task induced by nicotine withdrawal, inhibited the neuroinflammatory response, and prevented neurogenesis correlated with cognitive deficits (Saravia et al. 2019).

Moreover, a recent study demonstrated that the anxiolytic effect of CBD on chronically-stressed mice depends on hippocampal neurogenesis. This effect may be an outcome of the pro-neurogenic activity of CBD, which may facilitate endocannabinoid signaling (Fogaça et al. 2018). On the other hand, some recent scientific advances have determined whether meth abstinence, as opposed to meth exposure, affects the proliferation/survival of progenitors in the hippocampus (Recinto et al. 2012). The most recent findings suggest that meth abstinence increases the proliferation/survival of newly born progenitors in the hippocampus.

The increased survival of progenitors can lead to improved neurogenesis in prolonged abstinence following meth (or cocaine) self-administration (Noonan et al. 2008; Recinto et al. 2012). Conversely, a previous study in 2018 showed that meth administration following prolonged withdrawal decreased cell survival in rats (García-Cabrerizo et al. 2018). Many markers of differentiation and maturation, which are used to identify CBD-induced changes in the morphological and functional transition of adult hippocampal neurons, are inefficient in chronic meth exposure. Therefore, in this study, we aimed to determine whether $\mathrm{CBD}$ treatment can change the number of proliferating cells during an abstinence period among rats with chronic exposure to meth in the hippocampal DG. 


\section{Materials And Methods}

\section{Subjects}

Sixty adult male albino Wistar rats (12 weeks old), weighing 240 to $280 \mathrm{~g}$, were used in this study. The subjects were kept under standard laboratory conditions, that is, a 12:12 hour light/dark cycle (lights on at 07:00), with ad libitum access to laboratory chow and tap water. The animals were randomly divided into various experimental groups. They were habituated to the new environment and kept there for one week before the experimental procedures. We attempted to minimize the animals' suffering and only use the minimum number of rats necessary to produce authentic scientific data. The experiments were carried out from 09:00 am to 15:00 pm. All tests were performed according to the Guide for the Care and Use of Laboratory Animals (National Institutes of Health Publication No. 80-23, revised in 1996). The experimental method was approved by the Research and Ethics Committee of Iran University of Medical Sciences, Tehran, Iran.

\section{Drugs}

Methamphetamine hydrochloride (synthesized and analyzed in the Laboratory of Medicinal Chemistry, Faculty of Pharmacy, Baghiyatallah University of Medical Sciences, Tehran, Iran) was freshly diluted in normal saline and was then administered subcutaneously (SC). Afterward, CBD (supplied by Tocris Bioscience, UK) was dissolved in $10 \%$ dimethyl sulfoxide (DMSO), phosphate buffer solution $90 \%$; and a $5 \mu \mathrm{l}$ mixture was injected into the lateral cerebral ventricle.

\section{METH administration procedure}

Fresh METH was dissolved in a volume of $0.9 \%$ saline solution before each administration. Rats $(n=60)$ repeatedly received injections of the same dose (i.e. $2 \mathrm{mg} / \mathrm{kg}$ [SC], twice a day, for 10 consecutive days). The control group received SC injections of saline $(1 \mathrm{ml} / \mathrm{kg})$ similarly twice a day, for a 10-day period. Injections were applied at 09:00 and 15:00 O'clock (Razavi et al. 2020b).

\section{Surgery and Microinjection Procedures}

The subjects were anesthetized through an intraperitoneal injection of a mixture containing ketamine $(100 \mathrm{mg} / \mathrm{kg})$ and xylazine $(10 \mathrm{mg} / \mathrm{kg})$. The lateral cerebral ventricle involved stereotaxic coordinates as follows: $1.6 \mathrm{~mm}$ lateral and $0.5 \mathrm{~mm}$ posterior to bregma, $4.2 \mathrm{~mm}$ deep from the dura. A stainless steel screw was used to fix the guide cannula in the skull and dental acrylic cement. The rats were handled and allowed to recover from surgery within 5-7 days. The injection device contained a polyethylene tube (PE20) connected to a $5-\mu \mathrm{L}$ Hamilton syringe as well as a 30 -gauge needle with $11-\mathrm{mm}$ length at the tip. The syringe was filled with drug at appropriate volume, and the injection needle was then inserted through the guide cannula (10 mm).(Razavi et al. 2020a)

\section{Histological Assays}


On the 20th day of experiments, 4 rats from each group were randomly anesthetized and perfused transcardially with ice-cold PBS (0.1 M; $\mathrm{pH}=7.4)$ and also 4\% paraformaldehyde (PFA, Merck, Germany). The subject brains were isolated, post-fixed in PFA during the night, and then cryo-protected in $30 \%$ sucrose (Merck, Germany) for a 48 -hour period at a $4{ }^{\circ} \mathrm{C}$ temperature. The brains were subsequently inserted, frozen at an optimal cutting temperature (OCT) compound (Bio Optica, Italy, \#059801), and kept at $-80^{\circ} \mathrm{C}$. The frozen coronal sections were made ready through a cryostat apparatus (Sci Lab, English) along the hippocampus anterior-posterior axis (AP: -3.2 to $-4.2 \mathrm{~mm}$ relative to bregma). It was then placed on gelatin-coated slides. As for immunofluorescence (IF) staining, the sections with a thickness of $10 \mu \mathrm{m}$ were washed with PBS and antigen retrieval was applied through immersing the slides in a citrate buffer ( $\mathrm{pH}=6)$ for a 30 -minute period at $80^{\circ} \mathrm{C}$ waters-bath (Jiao et al. 1999). The sections were subsequently permeabilized through a volume of $1 \%$ Triton X-100, blocked and incubated during the night at $4{ }^{\circ} \mathrm{C}$ with several primary antibodies as follows: rabbit-anti Ki67 (1:100, Abcam, USA, \#ab66155), rabbit anti-DCX antibody (1:100, Abcam, USA, \#ab77450) and rabbit anti-NeuN antibody (1:100, Abcam, USA, \# ab104225). We detected the immunoreactivities through incubation with goat anti-rabbit IgG FITC conjugated antibody (1:50, Sigma, USA, \#F1262) for one hour at room temperature, which was followed by nuclear counterstaining with the DAPI re-agent (Sigma, USA). Images were subsequently obtained by a fluorescence microscope (manufactured by Olympus). NeuN, Ki67. The DCX-positive cells were counted within $\times 20$ microscopic fields in the hippocampus' SGZ region into minimum three sections per subject, using ImageJ. The count of positive cells was illustrated based on the percentage of total DAPI-stained cells (Chavoshinezhad et al. 2019; Daval et al. 2009; Vithlani et al. 2013).

The 10- $\mu$ m-thick sections were stained with Nissl according to the protocol that was previously reported (Miller et al. 2013). By defatting the sections in xylene, we hydrated them through decreasing concentrations of ethanol and water. They were then incubated in $0.3 \%$ Cresyl Violet solution (Sigma, USA) for five minutes at a temperature of $50^{\circ} \mathrm{C}$. By rinsing in water, the sections were dehydrated with incrementing concentrations of ethanol, cleared in xylene, cover-slipped and obtained by a Nikon light microscope. The cells in 3 sections of the dentate gyrus area of the hippocampus per animal were counted by Image $\mathrm{J}$ software v1.8 (NIH, Wayne Rasband, and USA). Cell shrinkage, loss of uniformity of Nissl body, cytoplasm, nucleus density, and pyknotic nucleus were evaluated in $1 \mathrm{~mm}$ area by a light microscope (Ooigawa et al. 2006).

\section{Experimental Design}

We examined the effects of repeated CBD administration on neurogenesis in rats with chronic exposure to meth during an abstinence period (Figure 1). There were four experimental groups treated with meth over a ten-day period (chronic exposure phase). One group of rats received only meth during the first ten days of chronic exposure. The abstinence protocol was applied for the other three groups. One group received $50 \mu \mathrm{g} / 5 \mu \mathrm{L}$ of CBD during the second ten days (abstinence period); the vehicle control group received $10 \%$ dimethyl sulfoxide (10\% DMSO+90\% PBS) instead of CBD as a vehicle during the abstinence period; and one group was the sham group. Also, two additional control groups (i.e., naïve) 
only received saline as the meth solvent, none during the induction and/or abstinence period. Every group included ten rats, and four rats from each group were randomly used in the histological assays.

\section{Statistical Analysis}

The data are all provided as mean \pm SEM. We conducted the one-way analysis of variance in order to compare the groups in differences of statistical significance levels. This was followed by Tukey's test for comparisons of multiple groups. The statistical analyses were entirely performed through GraphPad Prism (V5; GraphPad, San Diego, CA). Differences between groups were deemed significant wherever $\mathrm{P}<0.05$.

\section{Results}

CBD treatment enhanced hippocampal neurogenesis during the abstinence period in the chronic meth exposure rats.

Since the ICV administration of CBD $(50 \mu \mathrm{g} / 5 \mu \mathrm{L})$ could particularly prevent impairments in memory recognition among rats with chronic exposure to meth during the abstinence period (Razavi et al. 2020b), we aimed to determine whether $\mathrm{CBD}$ affected the expression of hippocampal neurogenesis markers, namely Ki67, NeuN, and DCX, which indicated the amount of neuronal proliferation and differentiation in the DG. Besides, immunofluorescence (IF) staining was carried out to measure the protein expression of neurogenesis markers in the hippocampus.

As illustrated in Fig. 2 and Fig. 3, one-way ANOVA, together with Tukey's post-hoc test $(P<0.001)$, indicated that Ki67-positive cells (as a proliferation marker) and DCX (a neuroblast marker) (Yuan et al. 2011b) significantly decreased in the treated meth-exposed group (black bar), as opposed to the salinetreated naive groups. Also, one-way ANOVA test, followed by Tukey's post hoc test, suggested that ki67and DCX-positive cells in subjects receiving CBD $(50 \mu \mathrm{g} / 5 \mu \mathrm{L})$ were significantly reversed during the abstinence period, compared to the naïve and saline control groups. Nonetheless, in ki67-positive cells, there were no significant differences between the CBD and control (DMSO) groups.

On the other hand, the difference in the number of DCX-positive cells was significant between the CBD group and the DMSO group $(P<0.05)$. These findings indicated that CBD could reverse ki67- and DCXpositive cells in the abstinence phase. Conversely, these cells (Ki67 and DCX) significantly increased in the DG (Fig. 2 \& Fig. $3 ; P<0.001)$ of the sham groups as compared to the meth group. Moreover, there was a significant difference between the CBD and sham groups in terms of the number of Ki-67- and DCX-positive cells $(P<0.001)$.

As shown in Fig. 4, the NeuN-positive cells, as opposed to Ki67- and DCX-positive cells, did not significantly increase in the CBD group, compared to the meth group in the DG. Conversely, one-way ANOVA demonstrated that the NeuN-positive cells significantly decreased in rats treated with meth as compared to the saline and control groups $(P<0.001)$. Moreover, there was a significant difference 
between the sham and CBD groups regarding the number of NeuN-positive cells $(P=0.004)$. However, the difference in the number of NeuN-positive cells was not significant between the CBD and DMSO groups $(P=0.225)$.

Neuronal degeneration in the DG of the hippocampus was diluted by CBD treatment in rats with chronic meth exposure

As shown in Fig. 5, there was a hippocampal neuronal loss in the meth group. One-way ANOVA test indicated that the percentage of dark neurons significantly reduced in the DG of the CBD-treated rat hippocampus as compared to the group with chronic exposure to meth $(P<0.001)$. Also, there was a significant increase in the percentage of dark neurons identified in the hippocampal subfield of the methexposede, compared to the saline and control groups (Fig. 5; $P<0.001$ ). Besides, there was a significant difference in the number of dark neurons between the CBD and sham groups $(P<0.001)$. However, the difference in the number of dark neurons was not significant between the CBD and DMSO groups $(P=$ $0.9)$. The results showed that $C B D$ administration in rats with chronic meth exposure significantly reduced the number of dark neurons in the hippocampal DG region $(P<0.001)$.

\section{Discussion}

The results of this study suggest that CBD has common neuroprotectant properties in rats with chronic meth exposure. Our findings indicated that repeated ICV administration of CBD at a dose of $50 \mu \mathrm{g} / 5 \mu \mathrm{L}$ led to higher cell proliferation (i.e., Ki-67 immunoreactivity) and increased the number of neurons (i.e., DCX immunoreactivity) in the hippocampal DG during an abstinence period in rats with chronic meth exposure. On the other hand, NeuN, as a neuron differentiation marker, did not show a significant increase in the CBD-treated group. We found that meth reduced the hippocampal cell proliferation, differentiation, and maturation, thereby increasing the rate of cell death. Moreover, we found that CBD could partially reverse meth-induced destruction.

A growing body of evidence suggests that adult hippocampal neurogenesis can be associated with the regulation of cognitive functions, whereas impaired neurogenesis has been found in psychiatric disorders, such as addiction, anxiety, and depression (David et al. 2010). We have recently identified cognitive deficits in behavioral tests, where impairments were detected in the spontaneous alternation behavior and recognition memory in the hippocampus. Besides, it has been shown that CBD could improve memory impairment in rats with chronic meth exposure (Razavi et al. 2020).

Researcher are increasingly suggesting that drug abuse can inhibit adult hippocampal neurogenesis, causing potentially adverse effects on cognitive regeneration and function (Eisch and Harburg 2006; Venkatesan et al. 2007); however, there are few studies on how meth affects neurogenesis. In a previous study, it was found that acute meth exposure in gerbils led to the lower proliferation of neuronal precursor cells within the hippocampal DG (Teuchert-Noodt et al. 2000). Also, lately, short-term administration of stimulant drugs, including meth, was associated with reduced neuronal precursor cell proliferation in the hippocampal SGZ of rats (Kochman et al. 2009). 
Additionally, rats with chronic or regular self-exposure to meth demonstrated a significant decrease in the hippocampal neurogenesis over several months (Mandyam et al. 2008). These studies indicated that meth intake could lead to reduced hippocampal neurogenesis and might play a role in cognitive dysfunction. It seems essential to clarify whether low doses of meth chronically affect the functional properties of immature and mature DG cells. In the current study, we investigated the effects of long-term (10 days), low-dosage meth administration ( $2 \mathrm{mg} / \mathrm{kg} / \mathrm{twice}$ per day) on the DG neurogenesis to evaluate changes in the synaptic plasticity of both immature and mature neurons.

These findings suggest that meth intake can cause a constant decrease in the number of neural stem cells; this may explain the psychostimulant-induced decline in hippocampal neurogenesis during drug use. Abstinence from meth exposure, however, leads to a higher net proliferation of progenitors and higher survival of newly born granular cells; therefore, the cell intrinsic signals, maintaining the stem cell pool and proliferation, are regulated differently during drug abstinence (Recinto et al. 2012). For example, an increase was observed in the count of DCX-positive cells in the DG of rats treated with CBD during the abstinence period; this marker of neuronal differentiation may be attenuated by meth (Deschaux et al. 2014).

Notably, Galinato et al. found that abstinence from meth increased the proliferation and differentiation of neural progenitors, while increasing adult neurogenesis in the DG (Galinato et al. 2018). It was found that a ten-day meth abstinence period increased the hippocampal neurogenesis markers in the treated groups. Consistent with our findings, Takashima and Mandyam (2018) observed an abstinence-induced increase in cell division, as well as aberrant survival of newly born progenitors as compared to normal control levels (Takashima and Mandyam 2018). These results agree with our findings, which revealed that the abstinence period might promote hippocampal neurogenesis in rats with chronic meth exposure.

On the other hand, Garcla-Cabrerizo et al. (2018) demonstrated the effects of meth administration on the hippocampal neurogenesis and found reinforced persistent adverse effects induced by meth in the rat hippocampus during prolonged withdrawal; also, they suggested that the immediate effects of meth administration on impaired cell proliferation during prolonged withdrawal led to the delayed production and decreased number of surviving cells; their results indicated the persistent effect of meth on neurogenesis regulation (García-Cabrerizo et al. 2018; Razavi et al. 2020b). Overall, these findings highlight the need for further studies. We believe that the expression of synaptic proteins and plasticity, promoting context-driven drug-related memories, essentially requires neurogenesis during abstinence.

Meanwhile, we determined all neuroplastic alterations due to ICV administration of CBD in rats chronically exposed to meth. It seems that a common result of meth exposure is the suppression of hippocampal neurogenic proliferation. Due to the lack of studies on the adult hippocampal neurogenesis in CBD-treated and meth-exposed mice, we aimed to determine whether the generally perceived reduction in the hippocampal neural proliferation could be possibly stimulated by CBD. We found a significant increase in Ki67/DCX staining in the DG of meth-exposed rats treated with CBD. Overall, evaluation of the 
effects of $C B D$ treatment and meth administration on the hippocampal neural proliferation suggests that $\mathrm{CBD}$ regulates the mechanisms through which meth diminishes the adult hippocampal neurogenesis.

So far, different drug-related anti-neurogenic mechanisms have been suggested as substitutes for mitochondrial dysfunction or oxidative stress (Cunha-Oliveira et al., 2008). CBD may cause more adult hippocampal neurogenesis, perhaps by intensifying the neurotransmission of CB1R in the hippocampus, as indicated in previous studies (Campos et al. 2013). Besides, we believe that these mechanisms are diverse due to their effects on adjusting hippocampal neuronal proliferation, as CBD treatment had a greater effect on changes in the Ki67/DCX/NeuN cell count compared to meth consumption, as shown by the statistical analysis. According to this hypothesis, CBD significantly reinforced the expression of CB1R in the hippocampus, a receptor that positively regulates adult neurogenesis in this area (Prenderville et al., 2015). While it seems impossible to determine how CBD causes these changes, earlier studies have shown comparable variations in the expression of CB1R following the administration of CBD (Ren et al., 2009; Viudez-Martínez et al., 2018).

It has been suggested that meth significantly increases the extracellular dopamine (Bennett et al., 1998) and serotonin (Rocher and Gardier, 2001) concentrations in the hippocampus, leading to chronic changes in the extracellular serotonin levels. Besides, stimulation of serotonin $1 \mathrm{~A}\left(5-\mathrm{HT}_{1 \mathrm{~A}}\right)$ receptors in the hippocampus can initiate neurogenesis. Therefore, by stimulating the long-term release of dopamine and serotonin, meth causes neurogenesis (Powrozek et al., 2004). Additionally, Zhang et al. (2016) found that activating $5-\mathrm{HT}_{1 \mathrm{~A}}$ receptors results in cAMP response element binding protein (CREB)-mediated neurogenesis; therefore, $\mathrm{CBD}$ plays a pro-neurogenic role by activating $5-\mathrm{HT}_{1 \mathrm{~A}}$ receptors (Russo et al., 2005; Zhang et al., 2016). In another study by Lujan M.Á et al. (2019), decreased cocaine intake induced by $C B D$ resulted in increased early neural differentiation/maturation, indicating the protective effect of this mechanism.

Although there is scarce information about the processes involved in modulating mesocorticolimbic signaling by neural proliferation and maturation adaptation, the available evidence can provide convincing data for elucidating this type of molecular mechanism (Luján et al., 2018). The meth-induced neurogenesis reduction can modify the protective effects of CBD. However, it seems inappropriate to assume that the mentioned mechanism is the only one influencing CBD in reducing chronic meth exposure in mice. It seems essential to conduct a thorough investigation about the modulation of dopaminergic signaling induced by $5-\mathrm{HT}_{1 \mathrm{~A}}$ receptors in the nucleus accumbens of mice receiving meth (Luján et al., 2018). The cognitive effects of CBD were detected at the same drug dosage, suggesting an effect on behavioral outcomes.

There is some evidence that cognitive performance may be associated with some forms of learning and memory-dependent hippocampal neurogenesis (for an extensive review, see Razavi et al., 2020). There have been consistent reports on the positive correlation of neurogenesis with cognitive performance. In the present study, for inducing chronic exposure in mice, they were exposed to $2 \mathrm{mg} / \mathrm{kg}$ of meth twice per day for ten days in novel object recognition and Y-maze tests; the results indicated the non-optimal 
cognitive performance. Since treatment with CBD showed neuroprotective effects, the recovered memory of rats treated with CBD might be associated with the pro-neurogenic effects. However, the present study did not address the explicit mechanistic effects of CBD on neurogenesis and cognitive responses; therefore, further studies are needed to elucidate the possible relationships (Razavi et al., 2020b).

In conclusion, the adult hippocampal neurogenesis is suppressed by the chronic administration of opiates (Eisch et al. 2000), alcohol (Nixon and Crews 2002), nicotine (Abrous et al. 2002), and cocaine (Yamaguchi et al. 2004). This study suggested that cannabinoids are the only substances that can enhance the adult hippocampal neurogenesis after chronic meth administration. A higher level of hippocampal neurogenesis appears to explain the neuroprotective effects of a chronic dose of CBD.

\section{Conclusion}

Our findings revealed that recurrent ICV administration of CBD could reinforce the expression of neurogenesis markers in a group of rats with chronic exposure to meth for an abstinence period. Besides, the behavioral effect of recurrent CBD administration is possibly mediated by enhancing cell proliferation/neurogenesis. This study supported the available evidence that suggests the capacity of $\mathrm{CBD}$ to modify operant behaviors related to meth. The findings also indicated the contribution of the proneurogenic effects of CBD to the recognition memory recovery. Overall, we provided some evidence about the functional effects of adult hippocampal neurogenesis on the potential reinforcement of meth. Since CBD can increase hippocampal neurogenesis, it can be proposed as an efficient tool to evaluate the effect of recently-generated hippocampal neurons on the neurobiology of meth dependence.

\section{Declarations}

\section{Data availability}

Available upon request.

\section{Conflict of interest}

The authors have declared no any conflict of interest exists.

\section{Ethical statement}

All experiments were done in accordance with the National Institutes of Health Guide for the Care and Use of Laboratory Animals (NIH Publication No. 80-23, revised 1996) and were approved by the Research and Ethics Committee of Iran University of Medical Sciences, Tehran, Iran (IR.IUMS.REC1395.27882).

\section{Acknowledgments}

This project was supported by the Vice-Chancellor for Research \& Technology of Iran University of Medical Sciences (grant No. 95011127882) for funding this work. Also, the authors would like to thank 
the Neuroscience Research Center, School of Medicine, Shahid Beheshti University of Medical Sciences.

\section{Role of Funding Sources}

Funding for this study was provided by the grant (No. 95111727882) from Vice-Chancellor for Research \& Technology of Iran University of Medical Sciences, Tehran, Iran. The Vice-Chancellor for Research \& Technology of Iran University of Medical Sciences had no further role in the design of the study; in the collection, analysis and interpretation of data; in the writing of the report; and in the decision to submit the paper for publication.

\section{Contributors}

Mehdi Mehdizadeh was responsible for the study concept and design. Yasaman Razavi contributed to the acquisition of data. Mehdi Mehdizadeh and Yasaman Razavi assisted with data analysis and interpretation of findings. Yasaman Razavi drafted the manuscript. Abbas Haghparast, Fariborz Keyhanfar and Ronak Shabani provided critical revision of the manuscript for important intellectual content. All authors critically reviewed content and approved final version for publication.

\section{References}

Abrous DN, Adriani W, Montaron MF, Aurousseau C, Rougon G, Le Moal M, Piazza PV (2002) Nicotine selfadministration impairs hippocampal plasticity J Neurosci 22:3656-3662 doi:20026324

Bennett BA, Hollingsworth CK, Martin RS, Harp JJ (1998) Methamphetamine-induced alterations in dopamine transporter function Brain Res 782:219-227 doi:10.1016/s0006-8993(97)01281-x

Campos AC et al. (2013) The anxiolytic effect of cannabidiol on chronically stressed mice depends on hippocampal neurogenesis: involvement of the endocannabinoid system International Journal of Neuropsychopharmacology 16:1407-1419

Canales JJ (2007) Adult neurogenesis and the memories of drug addiction European archives of psychiatry and clinical neuroscience 257:261-270

Canales JJ (2010a) Comparative neuroscience of stimulant-induced memory dysfunction: role for neurogenesis in the adult hippocampus Behav Pharmacol 21:379-393 doi:10.1097/FBP.0b013e32833e16b6

Canales JJ (2010b) Comparative neuroscience of stimulant-induced memory dysfunction: role for neurogenesis in the adult hippocampus Behavioural pharmacology 21:379-393

Chavoshinezhad S, Mohseni Kouchesfahani H, Ahmadiani A, Dargahi L (2019) Interferon beta ameliorates cognitive dysfunction in a rat model of Alzheimer's disease: Modulation of hippocampal neurogenesis and apoptosis as underlying mechanism Prog Neuropsychopharmacol Biol Psychiatry 94:109661 doi:10.1016/j.pnpbp.2019.109661 
Cunha-Oliveira T, Rego AC, Oliveira CR (2008) Cellular and molecular mechanisms involved in the neurotoxicity of opioid and psychostimulant drugs Brain research reviews 58:192-208

Daval JL, Blaise S, Gueant JL (2009) Vitamin B deficiency causes neural cell loss and cognitive impairment in the developing rat Proc Natl Acad Sci U S A 106:E1; author reply E2 doi:10.1073/pnas.0809217106

David DJ, Wang J, Samuels BA, Rainer Q, David I, Gardier AM, Hen R (2010) Implications of the functional integration of adult-born hippocampal neurons in anxiety-depression disorders The Neuroscientist 16:578-591

Deschaux 0 et al. (2014) Hippocampal neurogenesis protects against cocaine-primed relapse Addict Biol 19:562-574 doi:10.1111/adb.12019

Eisch AJ, Barrot M, Schad CA, Self DW, Nestler EJ (2000) Opiates inhibit neurogenesis in the adult rat hippocampus Proc Natl Acad Sci U S A 97:7579-7584 doi:10.1073/pnas.120552597

Eisch AJ, Harburg GC (2006) Opiates, psychostimulants, and adult hippocampal neurogenesis: Insights for addiction and stem cell biology Hippocampus 16:271-286

Fogaça MV, Campos AC, Coelho LD, Duman RS, Guimarães FS (2018) The anxiolytic effects of cannabidiol in chronically stressed mice are mediated by the endocannabinoid system: role of neurogenesis and dendritic remodeling Neuropharmacology 135:22-33

Galinato MH et al. (2018) Neurogenesis during abstinence is necessary for context-driven methamphetamine-related memory Journal of Neuroscience 38:2029-2042

García-Cabrerizo R, Bis-Humbert C, García-Fuster MJ (2018) Methamphetamine binge administration during late adolescence induced enduring hippocampal cell damage following prolonged withdrawal in rats Neurotoxicology 66:1-9

Gerdes J, Lemke H, Baisch H, Wacker H-H, Schwab U, Stein H (1984) Cell cycle analysis of a cell proliferation-associated human nuclear antigen defined by the monoclonal antibody Ki-67 The journal of immunology 133:1710-1715

Hildebrandt K, Teuchert-Noodt G, Dawirs R (1999) A single neonatal dose of methamphetamine suppresses dentate granule cell proliferation in adult gerbils which is restored to control values by acute doses of haloperidol Journal of neural transmission 106:549-558

Jessberger S et al. (2009) Dentate gyrus-specific knockdown of adult neurogenesis impairs spatial and object recognition memory in adult rats Learning \& memory 16:147-154

Jiao $Y$ et al. (1999) A simple and sensitive antigen retrieval method for free-floating and slide-mounted tissue sections Journal of neuroscience methods 93:149-162 
Jun H, Mohammed Qasim Hussaini S, Rigby MJ, Jang M-H (2012) Functional role of adult hippocampal neurogenesis as a therapeutic strategy for mental disorders Neural plasticity 2012

Kempermann G (2008) The neurogenic reserve hypothesis: what is adult hippocampal neurogenesis good for? Trends in neurosciences 31:163-169

Kim YT et al. (2010) Alterations in cortical activity of male methamphetamine abusers performing an empathy task: fMRI study Human Psychopharmacology: Clinical and Experimental 25:63-70

Kochman LJ, Fornal CA, Jacobs BL (2009) Suppression of hippocampal cell proliferation by short-term stimulant drug administration in adult rats Eur J Neurosci 29:2157-2165 doi:10.1111/j.14609568.2009.06759.x

Leuner B, Gould E, Shors TJ (2006) Is there a link between adult neurogenesis and learning? Hippocampus 16:216-224

Llorens-Martín M, Torres-Alemán I, Trejo JL (2006) Pronounced individual variation in the response to the stimulatory action of exercise on immature hippocampal neurons Hippocampus 16:480-490

Luján MÁ, Castro-Zavala A, Alegre-Zurano L, Valverde O (2018) Repeated Cannabidiol treatment reduces cocaine intake and modulates neural proliferation and CB1R expression in the mouse hippocampus Neuropharmacology 143:163-175

Mandyam CD, Wee S, Crawford EF, Eisch AJ, Richardson HN, Koob GF (2008) Varied access to intravenous methamphetamine self-administration differentially alters adult hippocampal neurogenesis Biological psychiatry 64:958-965

Miller JA et al. (2013) Conserved molecular signatures of neurogenesis in the hippocampal subgranular zone of rodents and primates Development 140:4633-4644

Nixon K, Crews FT (2002) Binge ethanol exposure decreases neurogenesis in adult rat hippocampus J Neurochem 83:1087-1093 doi:10.1046/j.1471-4159.2002.01214.x

Noonan MA, Choi KH, Self DW, Eisch AJ (2008) Withdrawal from cocaine self-administration normalizes deficits in proliferation and enhances maturity of adult-generated hippocampal neurons $\mathrm{J}$ Neurosci 28:2516-2526 doi:10.1523/jneurosci.4661-07.2008

Ooigawa H, Nawashiro H, Fukui S, Otani N, Osumi A, Toyooka T, Shima K (2006) The fate of Nissl-stained dark neurons following traumatic brain injury in rats: difference between neocortex and hippocampus regarding survival rate Acta neuropathologica 112:471-481

Parent JM (2003) Injury-induced neurogenesis in the adult mammalian brain The Neuroscientist 9:261272 
Potvin S, Stavro K, Rizkallah É, Pelletier J (2014) Cocaine and cognition: a systematic quantitative review Journal of addiction medicine 8:368-376

Powrozek TA, Sari Y, Singh RP, Zhou FC (2004) Neurotransmitters and substances of abuse: effects on adult neurogenesis Curr Neurovasc Res 1:251-260 doi:10.2174/1567202043362225

Prenderville JA, Kelly ÁM, Downer EJ (2015) The role of cannabinoids in adult neurogenesis British journal of pharmacology 172:3950-3963

Razavi Y, Shabani R, Mehdizadeh M, Haghparast A (2020a) Neuroprotective effect of chronic administration of cannabidiol during the abstinence period on methamphetamine-induced impairment of recognition memory in the rats Behav Pharmacol 31:385-396 doi:10.1097/fbp.0000000000000544

Razavi Y, Shabani R, Mehdizadeh M, Haghparast A (2020b) Neuroprotective effect of chronic administration of cannabidiol during the abstinence period on methamphetamine-induced impairment of recognition memory in the rats Behavioural Pharmacology

Recinto P et al. (2012) Levels of neural progenitors in the hippocampus predict memory impairment and relapse to drug seeking as a function of excessive methamphetamine self-administration Neuropsychopharmacology 37:1275-1287 doi:10.1038/npp.2011.315

Ren Y, Whittard J, Higuera-Matas A, Morris CV, Hurd YL (2009) Cannabidiol, a nonpsychotropic component of cannabis, inhibits cue-induced heroin seeking and normalizes discrete mesolimbic neuronal disturbances Journal of Neuroscience 29:14764-14769

Rezapour T, DeVito EE, Sofuoglu M, Ekhtiari H (2016) Perspectives on neurocognitive rehabilitation as an adjunct treatment for addictive disorders: from cognitive improvement to relapse prevention. In: Progress in brain research, vol 224. Elsevier, pp 345-369

Rocher C, Gardier AM (2001) Effects of repeated systemic administration of d-Fenfluramine on serotonin and glutamate release in rat ventral hippocampus: comparison with methamphetamine using in vivo microdialysis Naunyn Schmiedebergs Arch Pharmacol 363:422-428 doi:10.1007/s002100000381

Russo EB, Burnett A, Hall B, Parker KK (2005) Agonistic properties of cannabidiol at 5-HT1a receptors Neurochemical research 30:1037-1043

Santarelli L et al. (2003) Requirement of hippocampal neurogenesis for the behavioral effects of antidepressants science 301:805-809

Saravia R, Ten-Blanco M, Grande MT, Maldonado R, Berrendero F (2019) Anti-inflammatory agents for smoking cessation? Focus on cognitive deficits associated with nicotine withdrawal in male mice Brain, behavior, and immunity 75:228-239 
Shors TJ, Miesegaes G, Beylin A, Zhao M, Rydel T, Gould E (2001) Neurogenesis in the adult is involved in the formation of trace memories Nature 410:372-376

Sofuoglu M, DeVito EE, Waters AJ, Carroll KM (2016) Cognitive function as a transdiagnostic treatment target in stimulant use disorders Journal of dual diagnosis 12:90-106

Takashima Y, Mandyam CD (2018) The role of hippocampal adult neurogenesis in methamphetamine addiction Brain Plasticity 3:157-168

Teuchert-Noodt G, Dawirs R, Hildebrandt K (2000) Adult treatment with methamphetamine transiently decreases dentate granule cell proliferation in the gerbil hippocampus Journal of neural transmission 107:133-143

Van Praag H, Christie BR, Sejnowski TJ, Gage FH (1999) Running enhances neurogenesis, learning, and long-term potentiation in mice Proceedings of the National Academy of Sciences 96:13427-13431

Venkatesan A, Nath A, Ming G-I, Song H (2007) Adult hippocampal neurogenesis: regulation by HIV and drugs of abuse Cellular and molecular life sciences 64:2120-2132

Vithlani M et al. (2013) The ability of BDNF to modify neurogenesis and depressive-like behaviors is dependent upon phosphorylation of tyrosine residues 365/367 in the GABAA-receptor $\gamma 2$ subunit Journal of Neuroscience 33:15567-15577

Viudez-Martínez A, García-Gutiérrez MS, Navarrón CM, Morales-Calero MI, Navarrete F, Torres-Suárez Al, Manzanares J (2018) Cannabidiol reduces ethanol consumption, motivation and relapse in mice Addiction biology 23:154-164

Wenzel JM, Cheer JF (2018) Endocannabinoid Regulation of Reward and Reinforcement through Interaction with Dopamine and Endogenous Opioid Signaling Neuropsychopharmacology 43:103-115 doi:10.1038/npp.2017.126

Yamaguchi M et al. (2004) Repetitive cocaine administration decreases neurogenesis in adult rat hippocampus Ann N Y Acad Sci 1025:351-362 doi:10.1196/annals.1316.043

Yuan CJ, Quiocho JMD, Kim A, Wee S, Mandyam CD (2011a) Extended access methamphetamine decreases immature neurons in the hippocampus which results from loss and altered development of neural progenitors without altered dynamics of the S-phase of the cell cycle Pharmacology Biochemistry and Behavior 100:98-108

Yuan SH et al. (2011b) Cell-surface marker signatures for the isolation of neural stem cells, glia and neurons derived from human pluripotent stem cells PloS one 6

Zhang J, Cai CY, Wu HY, Zhu LJ, Luo CX, Zhu DY (2016) CREB-mediated synaptogenesis and neurogenesis is crucial for the role of 5-HT1 a receptors in modulating anxiety behaviors Sci Rep 6:29551 


\section{Figures}

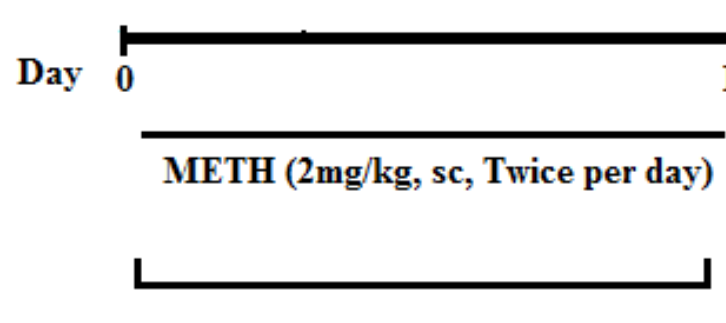

Chronic exposure phase
Histological assays
20

CBD $(50 \mu \mathrm{g} / 5 \mu \mathrm{l}, \mathrm{ICV}$, per day)

\section{Abstinence period}

Figure 1

Schematic illustration of the experimental protocol. For the induction of chronic exposure METH or vehicle, animals received METH/saline ( $2 \mathrm{mg} / \mathrm{kg}$, twice/day) for 10 days. Thereafter, the effect of ICV administration of CBD (50 $\mathrm{g} / 5 \mu \mathrm{l})$ or vehicle (DMSO) during the abstinence period (10-20 days). After completion of procedures animals were sacrificed and hippocampus tissues were removed for histological assays.

(a)
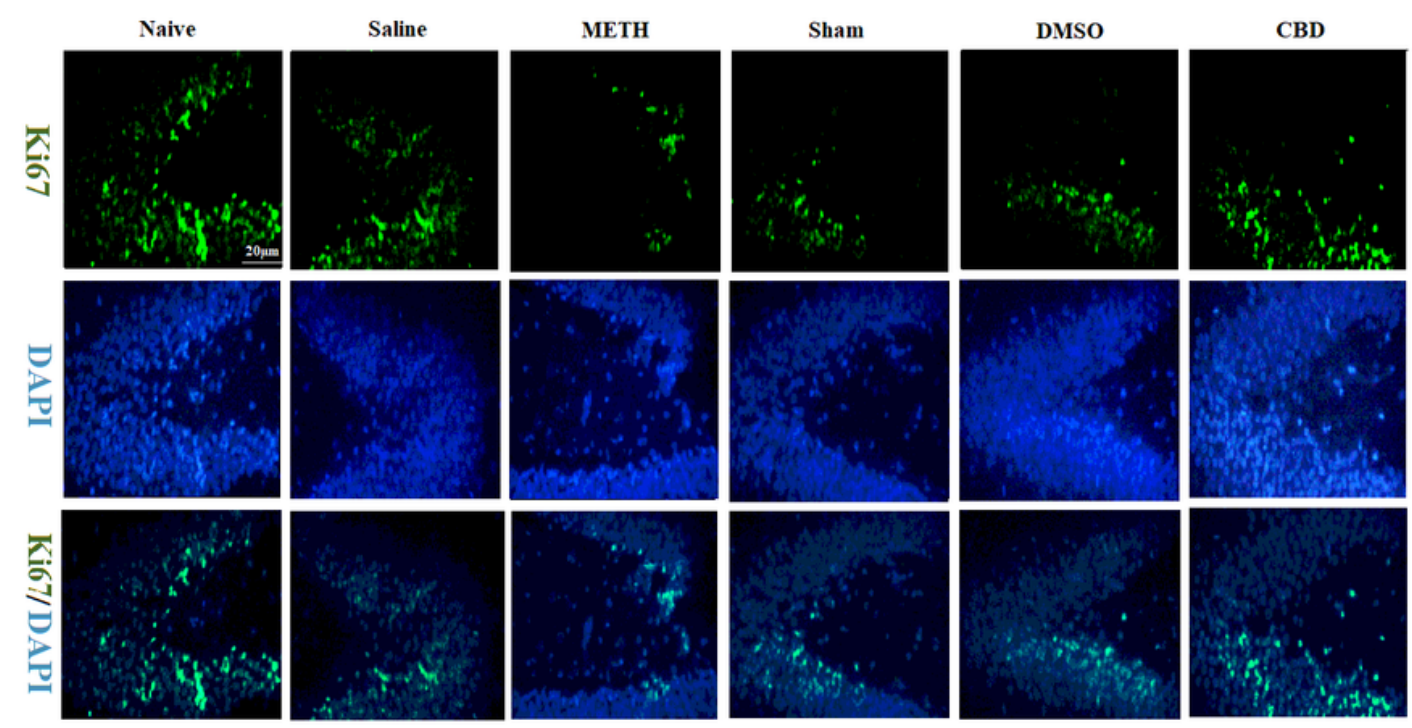

(b)
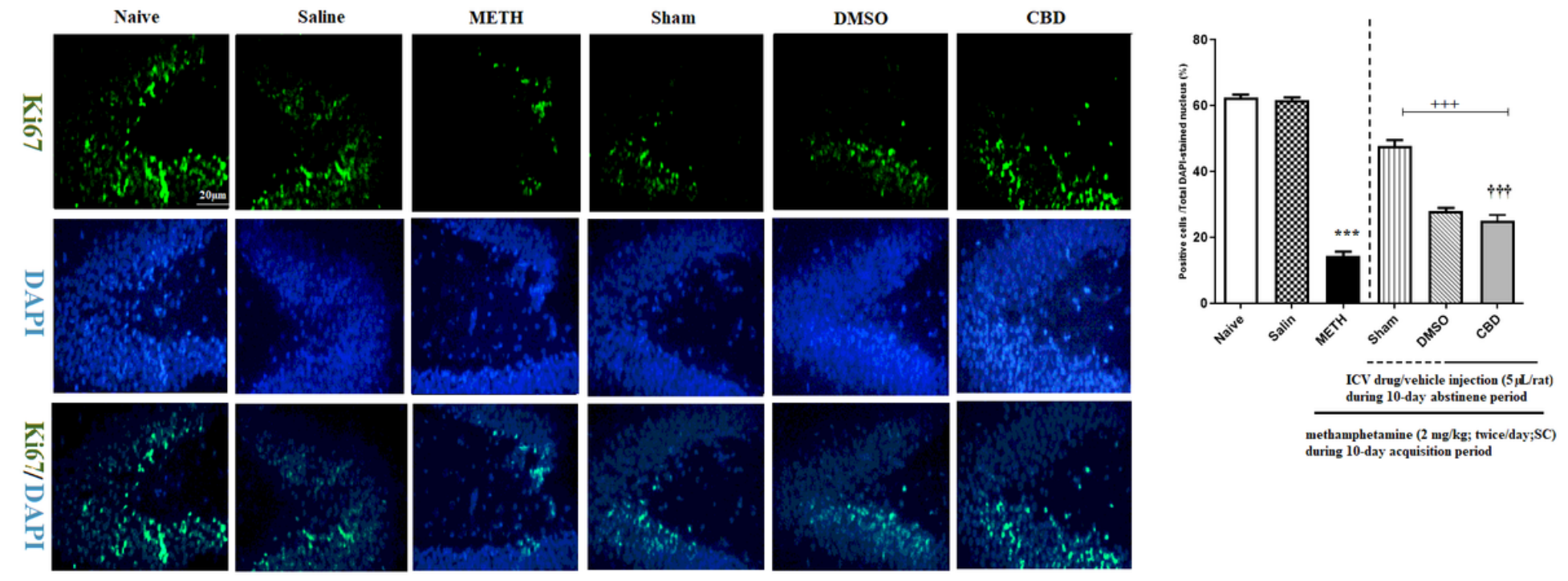

Figure 2

The effect of ICV administration of CBD on Ki67 positive cells in the hippocampal dentate gyrus in chronic exposure METH rats. Immunofluorescence images of Ki67 (green), DAPI (blue) and Ki67 /DAPI merged image in each group (a) Representative micrographs of immunofluorescence staining of Ki67 in Naïve (Control), Saline, Sham, METH, DMSO and CBD groups. Scale bar=20 $\mu \mathrm{m}$. The percentage of (b) Ki67 positive cells reported as the mean \pm SEM. $n=4$ /group; the differences between groups were 
determined by ANOVA followed by Tukey test. $\triangle \mathbb{Q P}<.001$ vs. the Naive group, \#\#\# < .001 vs. the METH group and $+++\mathrm{P}<.001$ vs. the Sham group

(a)

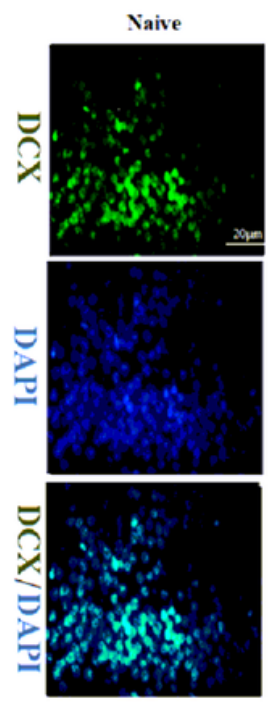

Saline

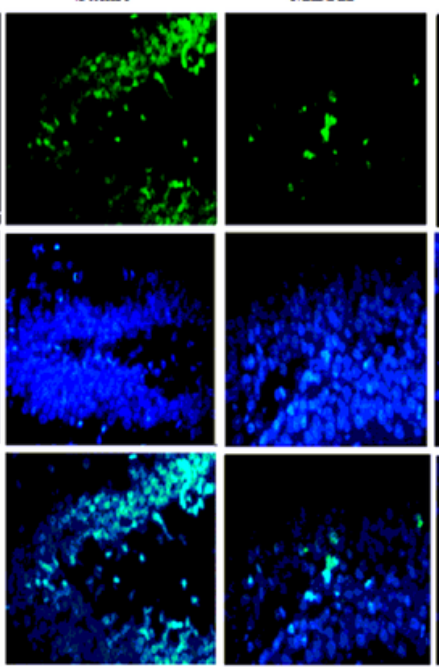

Sham

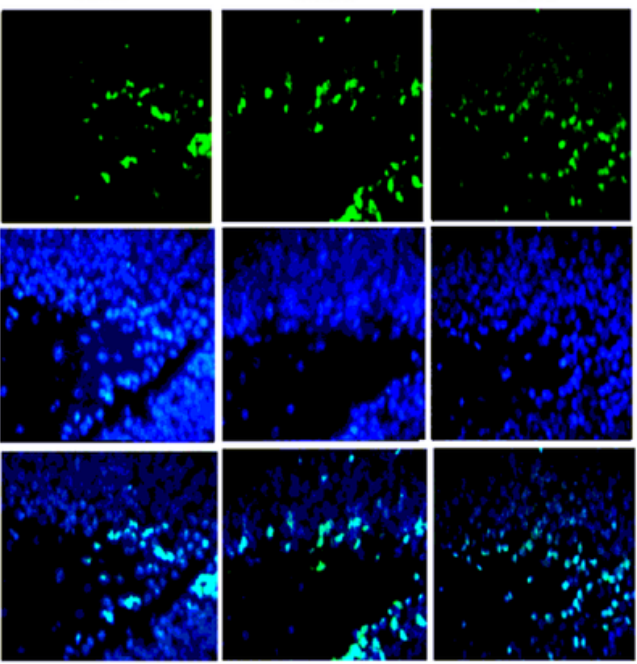

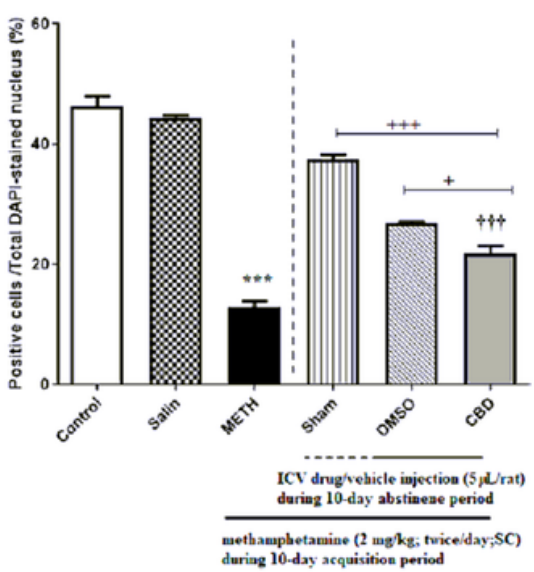

Figure 3

The effect of ICV administration of CBD on the protein expression of neurogenesis marker (DCX) in the dentate gyrus in chronic exposure METH rats (a) Representative micrographs of immunofluorescence staining of Doublecortin (DCX) in Naïve (Control), Saline, Sham, METH, DMSO and CBD groups. Cell nuclei were counterstained with DAPI. Scale bar=20 $\mu \mathrm{m}$. Immunofluorescence images of DAPI (blue), DCX (green) and D /DAPI merged image in each group The percentage of (b) Doublecortin (DCX) positive cells reported as the mean \pm SEM. $n=4$ /group; the differences between groups were determined by ANOVA followed by Tukey test. $\triangle \mathbb{Q} \otimes P<.001$ vs. the Naive group, \#\#\#P $<.001$ vs. the METH group, $+++P<.001$ vs. the Sham group and $+\mathrm{P}<0.05$ vs. the DMSO group

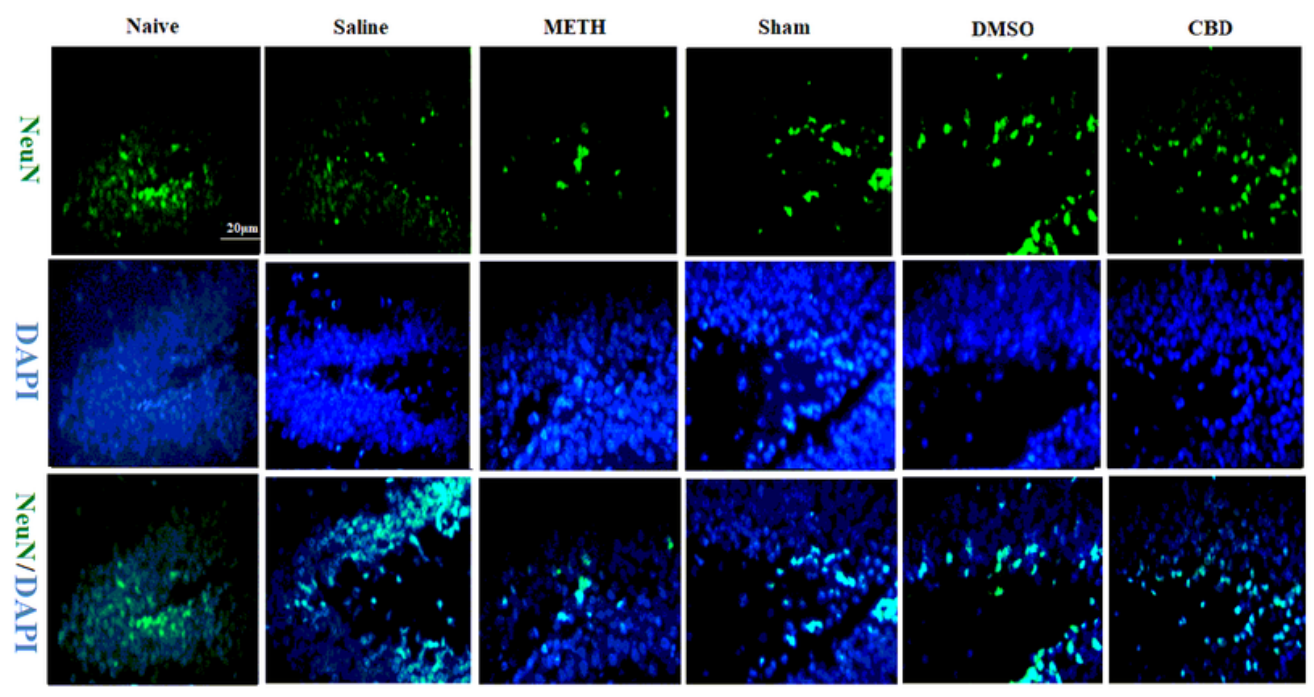

(b)

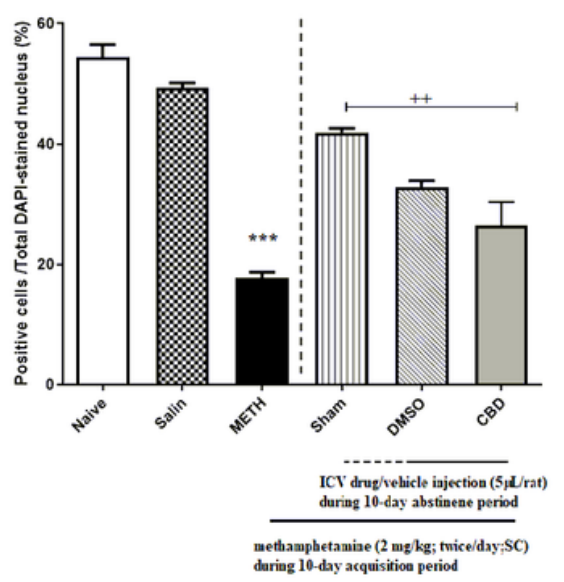

Figure 4 
Quantitative analysis of NeuN positive cells on ICV administration of CBD in hippocampal dentate gyrus of adult chronic exposure METH rats. (a) Representative micrographs of immunofluorescence staining of NeuN in Naïve (Control), Saline, Sham, METH, DMSO and CBD groups. Scale bar=20 $\mu \mathrm{m}$. Immunofluorescence images of DAPI (blue), NeuN (green) and NeuN /DAPI merged image in each group. The percentage of (b) NeuN positive cells reported as the mean \pm SEM. $n=4 /$ group; the differences between groups were determined by ANOVA followed by Tukey test. $\mathbb{X} \mathbb{Q} \mathrm{P}<.001$ vs. the Naive group, $++\mathrm{P}<$ .01 vs. the Sham group.

(a)

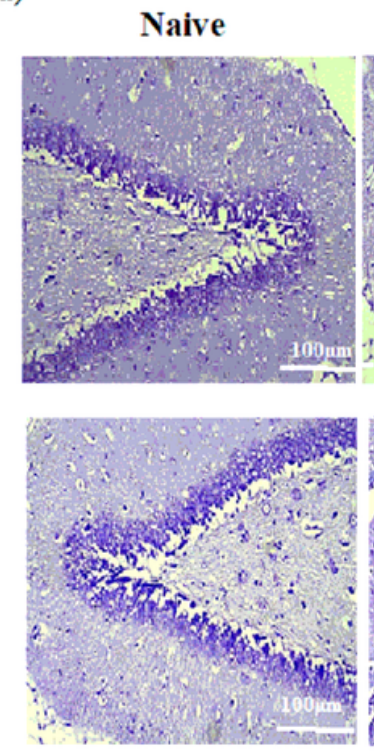

Sham
Saline
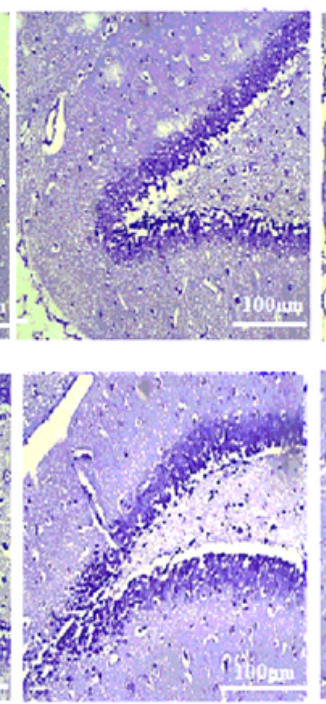

DMSO
METH
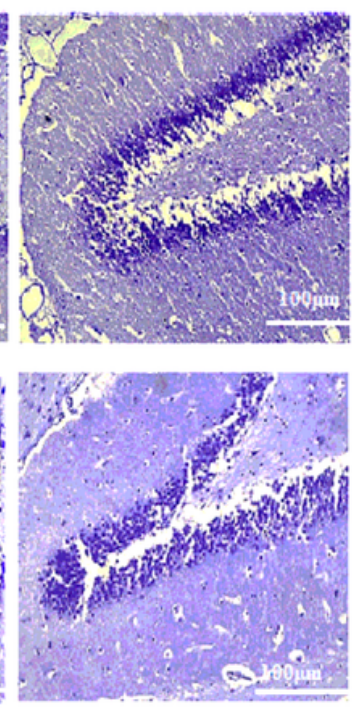

CBD (b)

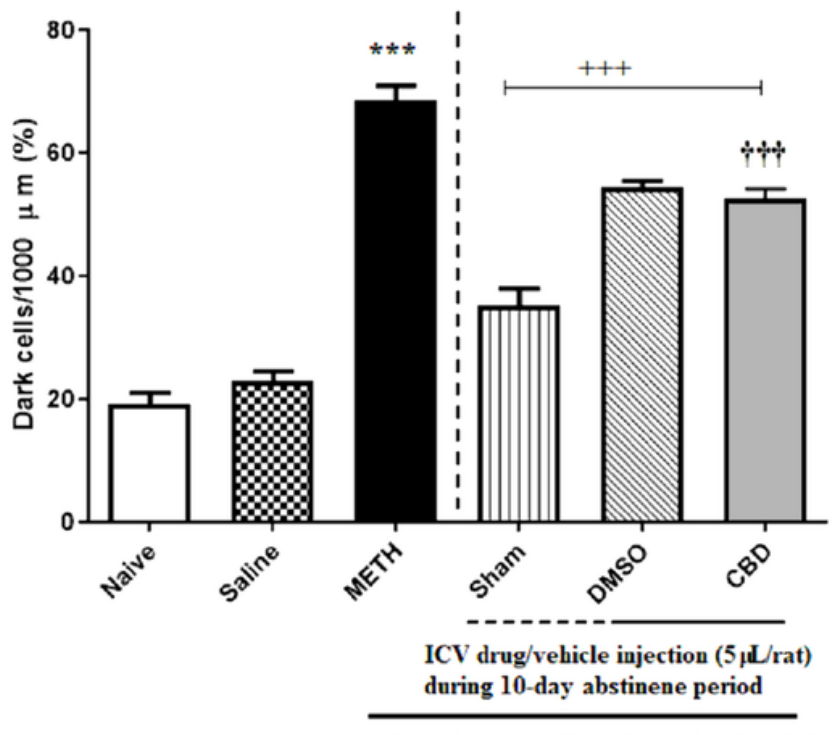

methamphetamine ( $2 \mathrm{mg} / \mathrm{kg}$; twice/day; $\mathrm{SC})$ during 10-day acquisition period

\section{Figure 5}

The effect of ICV administration of CBD on hippocampus neuron degeneration in the dentate gyrus in chronic exposure METH rats. (a) Representative micrographs of nissl staining of neuronal cells in the DG hippocampal subfield of the Naïve (Control), Saline, Sham, METH, DMSO and CBD groups, scale bar $=100 \mu \mathrm{m}$. (b) The percentage of dark neurons in the hippocampus. Data are reported as the mean \pm SEM. $n=4$ /group; the differences between groups were determined by ANOVA followed by Tukey test.

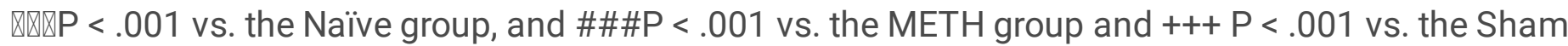
group. 